\title{
Epigenetics in Mammary Gland Biology and Cancer
}

\author{
Eve Devinoy • Monique Rijnkels
}

Published online: 11 February 2010

(C) Springer Science+Business Media, LLC 2010

In the post genome era, the focus has shifted to understanding the mechanisms that regulate the interpretation of the genetic code. "Epigenetics" as a research field is taking center stage. Epigenetics is a term which is now being used throughout the scientific community in different contexts from physiology to cell biology and genetics with diverse meanings inherited from the past [1].

The term "Epigenetic" was first coined in the nineteen forties by C.H. Waddington when he proposed the concept of the "Epigenetic landscape" for the process of cellular decision-making during development [2, 3]. He developed this concept by taking into account heterogeneous data from genetics, evolutionary biology, and embryology, providing a conceptual model of the interaction of genetic material with its surroundings to produce a phenotype.

Since then, the concept of epigenetics has evolved. It is now often defined as "the manifestation of a phenotype, which can be transmitted to the next generation of cells or individual, without alterations to the DNA sequence (genotype)". An epigenetic event is a cell's or organism's response to external queues, which become part of its developmental repertoire, modifying gene expression profiles without alterations to the underlying DNA sequences.

\footnotetext{
E. Devinoy $(\bowtie)$

INRA, UR1196, Génomique et Physiologie de la Lactation, 78352 Jouy en josas Cedex, France

e-mail: eve.devinoy@jouy.inra.fr

M. Rijnkels

Pediatrics-Nutrition, USDA/ARS Children's Nutrition

Research Center, Baylor College of Medicine,

1100 Bates Street,

Houston, TX 77030-2600, USA

e-mail: rijnkel@bcm.tmc.edu
}

Epigenetic states can be maintained through mitoses but also through meioses and be transmitted from one generation to the next.

In the animal kingdom, examples of epigenetic events transmitted from one generation to the next are rare [4]. In rats [5] or mice [6], nutrition has been shown to affect the phenotype of the offspring. Variability in the mouse Agouti phenotype (itself related to obesity and predisposition to diabetes) [7] has been reported in a homogeneous genetic background. A few examples of epigenetic inheritance in humans are still under debate such as grandchild phenotype which would depend on grandmother's or grandfathers food supply or smoking [8].

On the contrary several responses to environmental conditions such as nutrition or stress at early stages of life resulting in different phenotypes during adulthood have been reported. Animal cloning clearly established a maternal imprinting during fetal life which affects growth and health in adults. In humans, it has been shown that mothers exposed to starvation during pregnancy give birth to children who will be on average smaller as adults [9]. When exposed to endocrine disruptors, women will give birth to males which will exhibit severe sexual disorders [10]. After birth, during the first week of postnatal life, maternal care also influences neuroendocrine and behavioral stress responses throughout life [11]. The most spectacular example might be the different phenotypes of "Queen" or "worker" observed in honey bees, depending on the nutrition of the larvae [12].

Thus epigenetics can explain the phenotypic outcome of interactions between environment and genetics, but it can also be seen as the basis of cellular differentiation. In that sense, it explains that in a multi-cellular organism development generates a plethora of cell types with distinct functions and diverse but stable gene expression profiles despite the shared genotype (same DNA) of the cells. 
In the strictest sense of the word, epigenetic is anything "in addition" (epi) to the genetic information encoded in the DNA. At the molecular level, epigenetic events are related to the chromatin state. Chromatin functions as an indexing system for the genetic information, controlling its accessibility and readout. Consequently, it determines transcriptional competence of a genomic region with a particular chromatin state. Chromatin is composed of nucleosomes-DNA-protein complexes formed by a $147 \mathrm{nt}$ DNA stretch wrapped around histone protein octamer (dimers of H2A,H2B, H3 and H4). Distinct chromatin states are characterized by covalent and non-covalent modifications to the DNA and histones. DNA methylation at the 5-carbon position of cytosine-thus far described mostly on $\mathrm{CpG}$ sequences-, post translational modifications of histones - acetylation, methylation, ubiquitinylation, phosphorylation-, presence of histone variants and non-histone chromosomal proteins all influence chromatin status (see also Table 1). The different chromatin states are classified as: euchromatin-decondensed chromatin, which harbors genes that are or can be actively transcribed, or heterochromatin - highly compacted and silenced chromatin. Constitutive heterochromatin is permanently silenced, whereas facultative heterochromatin is transcriptionally repressed based on developmental stage, cell cycle or cellular location (Table 1).

Berger and colleagues recently proposed an elegant framework for epigenetic regulation [13]. The external signals received by the cell (or organism), such as developmental signals, nutrition, environmental exposures and disease triggers are called "epigenators". Cell shape, cellcell and cell-extracellular matrix interactions can also be considered as epigenators. This external signal is transduced by "epigenetic initiators" which determine time and place of epigenetic modifications. Some of these initiators are DNA binding factors and non-coding RNAs. "Epigenetic maintainers" are the enzymes and proteins that read, write and erase the various DNA and histone modifications. They govern the dynamic changes between the chromatin states and in doing so maintain or change the epigenetic state including the higher-order chromatin structure and possibly also nuclear organization (Fig. 1).

Based on this paradigm, we can see how both more direct transcriptional regulation and cellular memory regulation use similar mechanisms to change chromatin status, to affect gene transcription in the short-term or long-term.

Most of the development and functional differentiation in the mammary gland occurs after birth. During the developmental windows of puberty, pregnancy and involution, the gland undergoes profound morphological and functional changes. These changes are regarded as a succession of cell fate determinations and lineage commitments. Research in the past decades has elucidated numerous signaling pathways involved in establishing specific expression patterns and morphological changes. Because of its postnatal development and differentiation, the mammary gland is an excellent model to study epigenetic processes.

In the light of the increased interest in epigenetic processes in development and disease, we have attempted in this issue to give an overview of the current knowledge of epigenetics with respect to the mammary gland.

In this issue we have adopted a very broad definition of epigenetics. Contributions approach epigenetics from different perspectives: from the possible involvement of chromatin changes in setting developmental programs as well as direct transcriptional regulation, to the influence of external signals on gene expression, whether or not mediated through chromatin changes, and the different aspects of epigenetic events involved in breast cancer etiology.

Epigenetic processes play an important role in the normal homeostasis of stem and progenitor cells in tissues and disruption can result in cancer. Cancer is marked by changes in epigenetic status of the genome. DNA is globally

Table 1 Characteristics of different chromatin compartments in mammalian genome.

\begin{tabular}{|c|c|c|c|}
\hline Chromatin characteristic & $\begin{array}{l}\text { Euchromatin } \\
\text { (active genes) }\end{array}$ & $\begin{array}{l}\text { Constitutive heterochromatin } \\
\text { (repetitive DNA) }\end{array}$ & $\begin{array}{l}\text { Facultative heterochromatin } \\
\text { (silenced genes) }\end{array}$ \\
\hline CpG DNA methylation & Hypomethylation & Hypermethylation & Hypermethylation \\
\hline \multirow[t]{6}{*}{ Histone modification } & H3/H4 hyperacetylation & H3/H4 hypoacetylation & H3/H4 hypoacetylation \\
\hline & H3K4 di-methylation & & \\
\hline & H3K4 tri-methylation & & \\
\hline & H3K9 mono-methylation & H3K9 tri-methylation & H3K9 di-methylation \\
\hline & H3K27 mono-methylation & & H3K27 tri-methylation \\
\hline & H4K20 mono-methylation & H4K20 tri-methylation & \\
\hline General chromatin features & Open chromatin & $\begin{array}{l}\text { Compacted chromatin } \\
\text { Clustered centromeres Heterochromatic foci }\end{array}$ & Compacted chromatin \\
\hline Transcription & Active gene transcription & & No gene transcription \\
\hline
\end{tabular}

Adapted from [14-16]; Subset of histone modifications is listed (see also [15, 16]) 


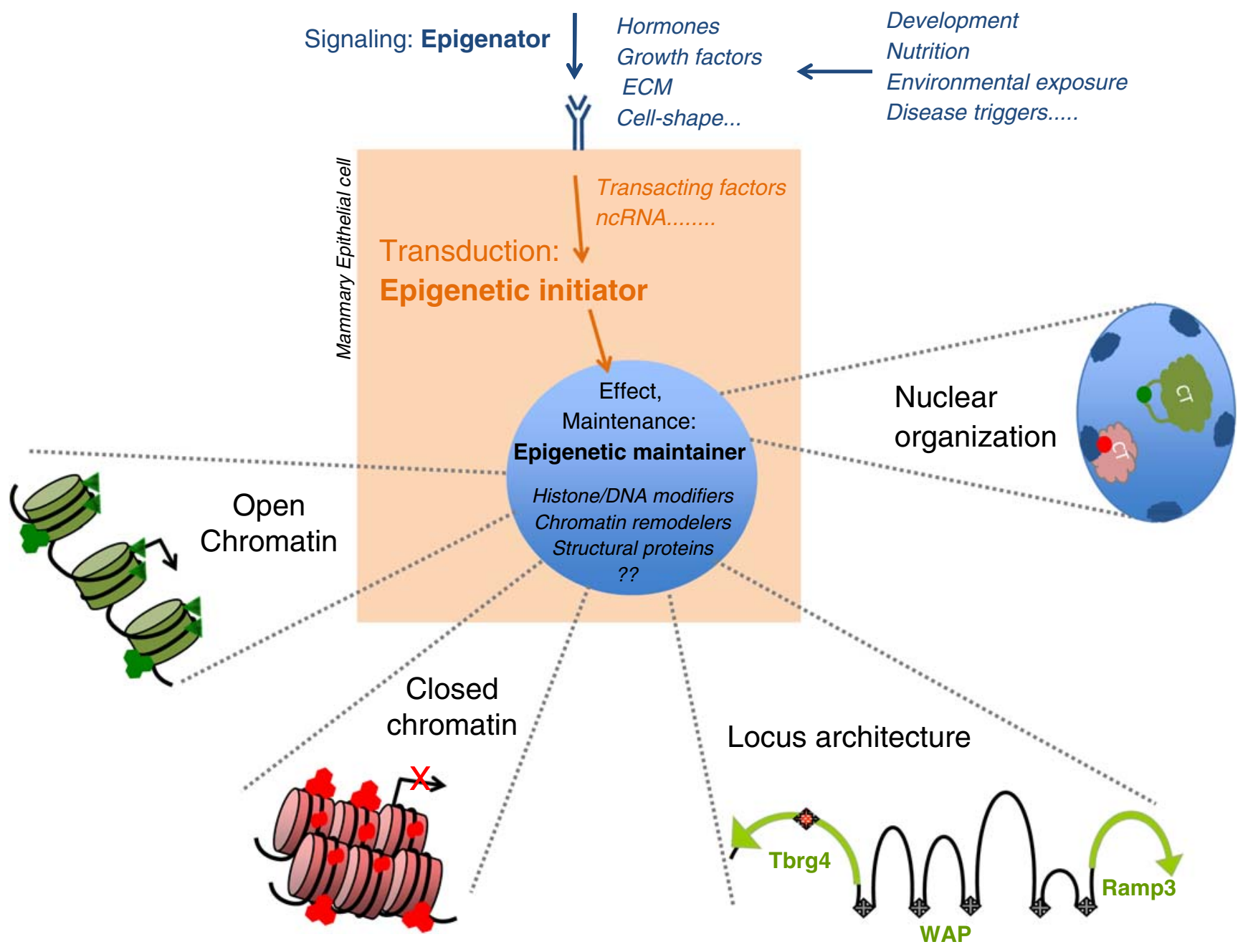

Figure 1 Summary of epigenetic regulation in mammary gland epithelium: Model is based on Berger and colleagues' paradigm for epigenetic regulation [13]. Signals from outside the cell, "epigenator" activate "epigenetic initiators" which signal to the "epigenetic maintainers" which modify the chromatin status and maintain it. Examples of epigenators in the mammary gland are: developmental signaling, nutrition, ECM- and Cell-Cell interactions, and Cell-shape. Epigenetic initiators: DNA binding factors, non-coding RNA (ncRNA). Epigenetic maintainers: histone and DNA modifying enzymes such as histone acetyl transferases (HAT), histone methyl transferases (HMT) DNA methyl transferases (DNMTS) etc., chromatin remodeling complexes, structural proteins such as HP1, Methyl binding proteins $(M B D, M E C P)$ etc., Histone variants. The resulting variations in chromatin, locus

hypomethylated, contributing to genome instability and activation of oncogenes, and locally hypermethylated causing silencing of tumor suppressor genes. There are also changes in histone modifications and chromatin organization. These aspects are discussed in the context of breast cancer in the contributions of Veeck et al., Dalvai et al. and Lelièvre. In breast cancer, DNA hypermethylation is induced by a stabilization of DNA methyltransferase transcripts as well as a decrease in microRNA targeting these transcripts (Veeck et al.). Specific histone modification profiles as well architecture and Nuclear organization are depicted. Histone modifications associated with open/accessible chromatin such as acetylation of Lysine residues in $\mathrm{H} 3$ or $\mathrm{H} 4$ are indicated as green triangles; histone modifications associated with closed/inactive chromatin (e.g. di- or trimethylation of lysine 9 in H3) are depicted as red hexagons, and DNA methylation is depicted as red ovals. Locus architecture is depicted as chromatin loop association with the nuclear matrix (black crosses) in lactogenic hormone induces MEC, (based on figure from Rijnkels et al. this issue). Nuclear organization is depicted with dark blue foci representing heterochromatic regions and colored regions labeled CT represent chromosome territories, associated with heterochromatin (red) or with a gene region looping out (green)(often associated with active or poised genes) see also Kress et al. this issue. as histone variants are also detected (Dalvai et al.; Veeck et al.). These epigenetic modifications may implicate altered estrogen receptor transduction pathways (Dalvai et al.; Pathiraja et al.) and explain endocrine resistance (Pathiraja et al.). The improvement of our knowledge in epigenetics of the mammary gland biology may therefore open new strategies in breast cancer therapy especially with respect to endocrine treatments (Pathiraja et al.).

As outlined above, alterations in the cellular phenotype during normal differentiation or development of organs 
might be considered as epigenetic events. In the mammary gland, which develops from fetal life to adulthood, and then undergoes successive cycles of development and regression, such epigenetic events have been identified around major milk protein genes (Rijnkels et al.) but most likely also occur at many other genes with changes in expression during mammary gland differentiation. Genome wide analysis will be needed to shed light on this. Epigenetic regulations may also explain phenotypic variations in dairy cows, which are unexplained by genetic polymorphisms and could be due to environmental conditions or disease (Singh et al.). Furthermore, in the mammary epithelial cell during lactation, the expression of milk protein genes, which carry epigenetic marks positive for transcription, is related to specific nuclear localization of these genes relative to their chromosome territories (Kress et al.).

In mammary tissue, the tight interactions of epithelial cells with their environment- the extracellular matrix and neighboring cells-influence normal cell growth, tissue specific gene expression and mammary cell homeostasis through epigenetic modifications (Spencer et al.; Lelièvre et al.) associated with specific 3D nuclear organization (Spencer, Lelièvre). Alterations of these interactions are related to breast cancer (Spencer et al.; Lelièvre et al.).

\section{Concluding Remarks}

Some of the remaining questions to be answered in the field of epigenetics are: how exactly are epigenetic marks targeted to specific genomic locations? and what are the signaling pathways that transduce such signals?

A number of "epigenators" modifying mammary cell fate can be identified. They can induce variation in chromatin conformation, which plays a key role in transcriptional regulation and underlies the epigenetic regulation during development and differentiation of the mammary gland. They might also play a role in the differentiation of mammary progenitors in different MEC populations. Aberrations in their signaling can induce changes in chromatin conformation and nuclear reorganization which can eventually lead to breast cancer. However, the signals that mediate these epigenetic changes are not well known yet.
Research in the laboratories contributing to this issue and several others is increasing our knowledge on this subject. We are only at the beginning, expanding efforts in this field will in the near future undoubtedly provide us with many new insights into the role of epigenetic regulation in mammary gland development and disease.

\section{References}

1. Jablonka E, Lamb MJ. The changing concept of epigenetics. Ann N Y Acad Sci. 2002;981:82-96.

2. Waddington $\mathrm{CH}$. Organisers and genes. Cambridge: Cambridge University Press; 1940.

3. Waddington CH. Strategy of the genes. London: Allen \& Unwin; 1957.

4. Morgan DK, Whitelaw E. The case for transgenerational epigenetic inheritance in humans. Mamm Genome. 2008;19:394-7.

5. Portha B. Programmed disorders of beta-cell development and function as one cause for type 2 diabetes? The GK rat paradigm. Diabetes Metab Res Rev. 2005;21:495-504.

6. Rakyan VK, Preis J, Morgan HD, Whitelaw E. The marks, mechanisms and memory of epigenetic states in mammals. Biochem J. 2001;356:1-10.

7. Morgan HD, Sutherland HG, Martin DI, Whitelaw E. Epigenetic inheritance at the agouti locus in the mouse. Nat Genet. 1999;23:314-8.

8. Pembrey ME, Bygren LO, Kaati G, et al. Sex-specific, male-line transgenerational responses in humans. Eur J Hum Genet. 2006;14:159-66.

9. Lumey LH. Decreased birthweights in infants after maternal in utero exposure to the Dutch famine of 1944-1945. Paediatr Perinat Epidemiol. 1992;6:240-53.

10. Godmann M, Lambrot R, Kimmins S. The dynamic epigenetic program in male germ cells: its role in spermatogenesis, testis cancer, and its response to the environment. Microsc Res Tech. 2009;72:603-19.

11. Weaver IC. Shaping adult phenotypes through early life environments. Birth Defects Res C Embryo Today. 2009;87:314-26.

12. Omholt SW, Amdam GV. Epigenetic regulation of aging in honeybee workers. Sci Aging Knowl Environ. 2004;2004:pe28.

13. Berger SL, Kouzarides T, Shiekhattar R, Shilatifard A. An operational definition of epigenetics. Genes Dev. 2009;23:781-3.

14. Arney KL, Fisher AG. Epigenetic aspects of differentiation. J Cell Sci. 2004;117:4355-63.

15. Rosenfeld JA, Wang Z, Schones DE, Zhao K, DeSalle R, Zhang MQ. Determination of enriched histone modifications in non-genic portions of the human genome. BMC Genomics. 2009;10:143.

16. Wang Z, Schones DE, Zhao K. Characterization of human epigenomes. Curr Opin Genet Dev. 2009;19:127-34. 